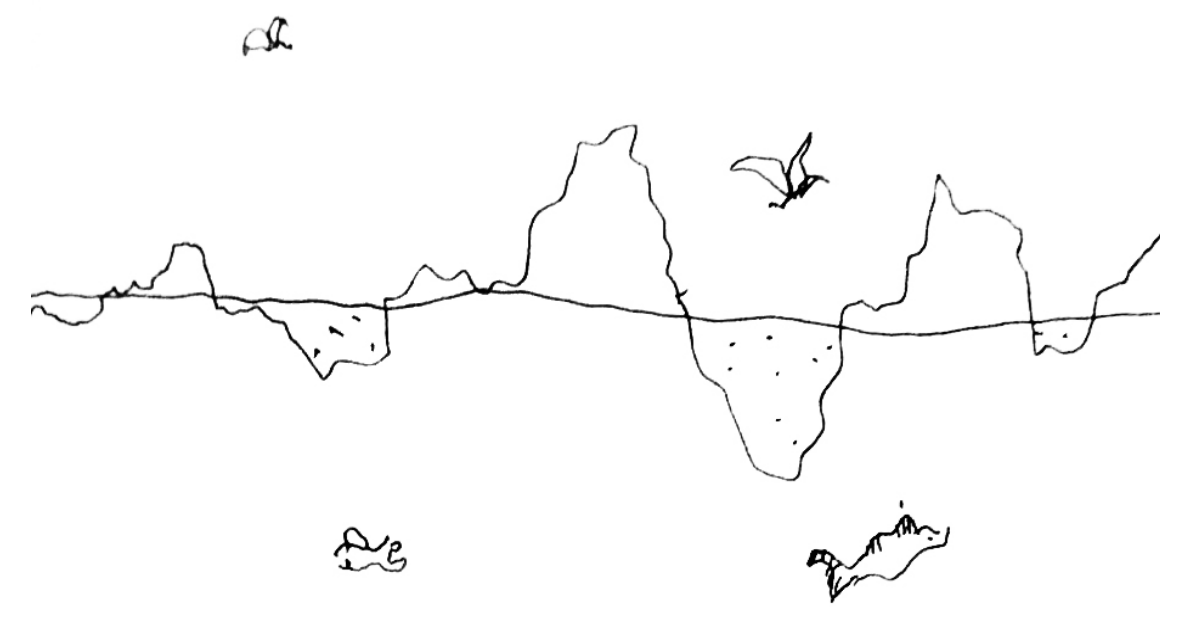

The fish and the bird

FIG. 1. Sverre Fehn, The fish and the bird, 1982. Tinta negra sobre papel,

$16,8 \times 20,7 \mathrm{~cm}$. Nasjonalmuseet Arkitektur, Oslo

\title{
De cómo distintos mecanismos de proyecto son capaces de construir el paisaje
}

\section{Joan Casals Pañella}

Recibido 2020.03.05 ::: Aceptado 2020.05.05

DOI: 10.5821/palimpsesto.21.9221

Persona de contacto: csls@coac.net

Doctor arquitecto por la UPC

\section{ABSTRACT}

Nuestra profesión sostiene un acuerdo con la ley de la actualmente permiten los avances de la ingeniería, consiguen salvar la dependencia del proyecto respecto del suelo. Conviene pronto asumir esta condición de la arquitectura, pues asumido el reto y aceptado el compromiso, fructíferas coyunturas aparecerán ante nosotros. En realidad, un dilema entre multitud de variables; como las que emanan de abundantes aspectos socio-fisicos, que definen cada de su propia experiencia vivida. Pero también, un dilema abierto entre notables constantes; como los mecanismos proyectuales que se dirigen a fundar la cota cero de la arquitectura, tomando mas o menos distancia respecto de una línea de horizonte, que al observarla con detenimiento, em de sus capas y de la relación que existe entre sus extremos y de hecho, en su famoso dibujo del pez y el pájaro, definio y de hecho, en su famoso dibujo del pez y el pajaro, definio tarde, explicaría además qué posibles relaciones posicionales podía tomar la arquitectura cuando dialoga con este soporte limítrofe. Aceptando ahora este posible diálogo alrededor de las fronteras de nuestros ineludibles cimientos, este artículo,
propone tomar aquí algunos ejemplos, para demostrar propone tomar aquí algunos ejemplos, para demostrar por Holl bajo la idea de Fehn, pueden además esconder un interés por construir el paisaje. Algunos fragmentos extraídos de la Casa Ugalde y La Ricarda por un lado, y el Parc Cementiri de lgualada y el Parc de Pedra Tosca, por otro lado, se prestan a esclarecer estas cuestiones. Y es que, alrededor de la cota cero y de unos excepcionales grados de ficción que definen el caracter individual de cada intervención, estos podios elevados o incluso generar nuevas topografías elevadas y semienterradas, como sistemas capaces de producir nuevos significados en el paisaje.

PALABRAS CLAVE: mecanismos; proyecto; suelo; límite; arquitectura; paisaje; ficción.
$\mathrm{N}$

uestra profesión sostiene un acuerdo con la ey de la gravedad, que ni los posibles desafíos estructurales que actualmente permiten

los avances de la ingeniería, consiguen salvar la dependencia del proyecto respecto del suelo1. Conviene pronto asumir esta condición de la arquitectura, pues asumido el reto y aceptado el compromiso, fructíferas coyunturas aparecerán ante nosotros.

En realidad, un dilema entre multitud de variables; como las que emanan de abundantes aspectos socio-físicos, que definen cada lugar concreto y que cada arquitecto interpreta a través de su propia experiencia vivida². Pero también, un dilema abierto entre notables constantes; como los mecanismos proyectuales que se dirigen a fundar la cota cero de la arquitectura, tomando más o menos distancia respecto de una línea de horizonte, que al observarla con detenimiento, emana realmente complejidad.

Sverre Fehn ${ }^{3}$, a propósito de estas cuestiones, tomó consciencia de su grueso, de sus capas y de la relación que existe entre sus extremos. De hecho, en uno de sus famosos dibujos, como el titulado "the fish and the bird", teniendo en cuenta algunas ideas de Heidegger ${ }^{4}$, definió esta idea de horizonte como un estado límite; frontera donde la arquitectura, como consecuencia de haber sido, tiene una nueva posibilidad de ser. El espacio-tiempo, por excelencia, de la invención arquitectónica. Quizás por ello, un elemento tantas veces dibujado por este sabio maestro nórdico.
Steven Holl, años más tarde, asumiendo la importancia de esta idea de horizonte para la arquitectura, explicaría además qué posibles relaciones posicionales podía llegar a tomar una construcción cualquiera cuando dialoga con su soporte limítrofe ${ }^{5}$. Inicialmente, estableció las a priori cuatro únicas posibilidades que parece que existen: bajo el suelo o enterrado, en el suelo o semienterrado, sobre el suelo o apoyado y por encima del suelo o a una determinada altura. No conforme con estas posiciones básicas, inmediatamente dedujo once posibles relaciones elementales más. Al final, acabó sumando veinticuatro nuevas posiciones. Las denominó complejas. Podrían haber sido más. En realidad, tratando de definir la posición a tomar por la arquitectura ante la presencia de un límite, no pudo llegar a describirlo. Pues, necesariamente invitado a lo indecidible con la idea de intermedio desarrollada por Merleau-Ponty 6 ; en la voz de este arquitecto fenomenológico, apareció de nuevo el ser heideggeriano, mostrando cómo todos los elementos individuales pierden sus fronteras cuando quedan abrazados en un campo común.

Ahora bien, aceptado este posible diálogo alrededor de las fronteras de nuestros ineludibles cimientos, no sólo es posible advertir el presumible dilatado juego proyectual anunciado, sino también la oportunidad de descubrir a través del mismo, cómo además estos mismos mecanismos de proyecto son capaces de enfocarse simultáneamente a construir el paisaje ; a relacionarse productivamente con el paisaje, y el paisaje con la arquitectura. En definitiva, un acto fundacional que la arquitectura establece con su anclaje; una acción en la que la arquitectura alcanza su mayor mérito poniendo en valor los fenómenos físicos y temporales de un lugar para devenir paisaje, pero también una acción por la que el paisaje, simultáneamente, resulta ser a la vez el conocimiento esencial constitutivo de la arquitectura. El momento cumbre en el que la ficción ${ }^{8}$ hace acto de presencia. Pues la posición, la tantas veces indecidible ubicación que puede tomar la arquitectura, también contribuye a poner en intriga; a recomponer, en concreto, a través de la verosimilitud, los estratos profundos de las historias que siempre preceden ante el porvenir posible de nuevas historias. Historias contenidas dentro de historias, encajes dentro de encajes, que afortunadamente, algunos fragmentos extraídos de la Casa Ugalde y La Ricarda por un lado, y el Parc de Pedra Tosca y el Parc Cementiri de Igualada, por otro lado, se prestan a explicar. Y es que, alrededor de la cota cero y de unos excepcionales grados de ficción que definen el carácter individual de cada intervención, estos ejemplos proponen aprovechar estratos existentes, construir podios elevados o incluso generar nuevas topografías elevadas y semienterradas, como sistemas capaces de producir nuevos significados en el paisaje.

\section{Sobre y en el suelo}

El primer análisis examina el muro de piedra encalado en blanco de $70 \mathrm{~m}$ de longitud, que Coderch proyectó para adentrarse en el interior de la Casa Ugalde desde el exterior de la parcela. Observando la obra que realmente fue construida, fácilmente puede advertirse cómo durante la fase de ejecución el muro prácticamente fue acortado a la mitad, propiciando como consecuencia un cambio en la aproximación prevista. Inicialmente, la idea era llegar, dejar el coche y caminar acompañado del muro hacia el interior de la casa. Pero, a partir de este cambio, para alcanzar primero el muro y luego la casa, empezó a ser necesario salvar un desnivel mediante el uso de unos escalones ubicados en la parte inferior media de la parcela. Desde este nuevo punto de partida, el muro unido en una continuidad perfecta a lo que se insinúa como un templo de color blanco, aparece conquistando la parte superior de una colina que domina unas vistas hasta entonces inadvertidas. En cierta manera, el muro está consiguiendo recrear lo que ya ofrecían las playas de Caldetes: el advertimiento de una arquitectura posada "sobre el suelo", que en cierta posición de dominio, se postula interesada en hacer de la construcción un elemento capaz de dotar al territorio de una nueva temporalidad; un elemento capaz de construir un paisaje, en este caso, como nuevo símbolo de salud, ocio y libertad. Ahora bien, superados los escalones necesariamente dispuestos para salvar el desnivel, Coderch nos ubica también en la base del muro. Aquí, un camino sumergido en un bosque de pinos, casi de forma inmediata, da cuenta de la función de contención que en efecto igualmente tiene destinado este elemento constructivo. El hecho posibilita reconocer que la arquitectura, si bien ha sido dispuesta "sobre el suelo", también lo ha sido simultáneamente "en el suelo". Es más, el muro construido, ya no solo invita a adentrarse a la casa como sugería el proyecto inicial. Desde esta nueva posición, parece querer interrogar a su visitante sobre la posibilidad de adentrarse hacia los terrenos colindantes. Aparece así el recuerdo de los muros de piedra seca 

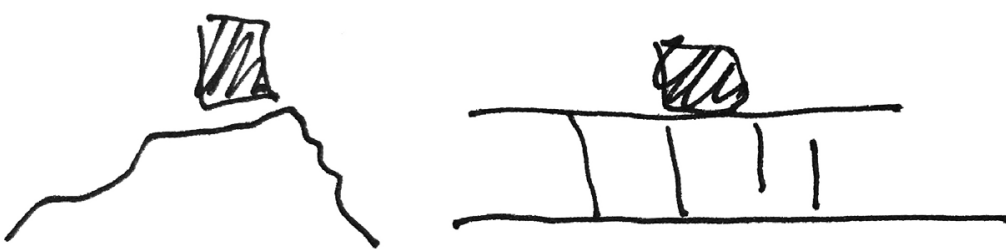

FIG. 2 Posiciones estudiadas. Autor

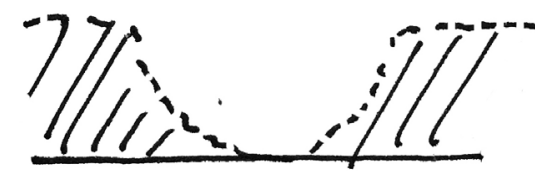

Tiłr, de los bancales, de las terrazas o de los márgenes del campo, haciendo reconocibles las circunstancias específicas del lugar, contrapuestos a la presencia de un artificioso encalado y a la evidencia del uso de argamasas. La sutileza a la que Coderch recurre para explicar que ,haciendo verosímil la arquitectura con el lugar, de una forma productiva e independiente, las dimensiones sociofísicas del paisaje se convierten simultáneamente en el conocimiento esencial constitutivo de la arquitectura.

\section{Sobre, pero por encima del suelo}

El segundo análisis, pone el foco en la plataforma horizontal de $8000 \mathrm{~m}^{2}$, que Bonet proyectó casi dos metros "por encima del suelo" existente en La Ricarda. Atendiendo al detalle de este elemento constructivo, puede verse un sistema de zapatas, jabalcones y vigas riostras de hormigón imponiendo un nuevo orden enfocado a domesticar la condición salvaje del lugar. La superficie de césped de aspecto recién cortado, contrastan realmente con el primitivismo que manifiesta el conjunto de plantas monocárpicas, escondidas entre unas dunas casi vírgenes, que tan bien congela Moisés Villelia, reutilizando tubos de fibrocemento provenientes de residuos industriales. Ahora bien, elevar la casa para prevenir a los futuros habitantes de los abundantes e históricos problemas de inundaciones, que desde tiempos remotos habían convivido en el lugar, demuestra que la plataforma no es una imposición caprichosa de una nueva temporalidad que se le exige al emplazamiento, sino más bien una posible respuesta a un conflicto. En la primera versión que Bonet hizo sobre la casa también elevó su planta baja dejando visto en aquella ocasión el sistema empleado para conseguirlo. Pero una vez presentó el proyecto, fue emplazado a pensar una casa que, además de estar más sumergida en el bosque, permitiera disfrutar de una nueva vida al aire libre como extensión del interior de la casa. Para conseguir estas proposiciones, Bonet pensó que debía mostrar la casa como si estuviera "sobre el suelo" aunque por cuestiones hidrológicas, tuviera que estar "por encima del suelo". Para conseguirlo, propuso construir un paisaje artificial elevado que, siendo imposible determinar en un primer instante su origen artificial, estableciera primero un específico diálogo con la casa, para después hacer lo mismo con la sustancia elemental del emplazamiento. Puede entenderse así, por ejemplo, el por qué de la dimensión de la plataforma y su consciente desmesura. Lejos está de querer enfocarse a que alguien pueda adivinar, en un primer encuentro, su artificial origen. En la misma dirección cabrá entender la insistencia en la dimensión horizontal del lugar; visible, por ejemplo, en los elementos constructivos que formalizan una cubierta suspendida sobre unos pilares o soportes, que, pintados de negro, desaparecen haciendo flotar el sistema. O en la voluntaria invasión de la naturaleza; ocurre, por ejemplo, de forma muy clara en el pasillo que da acceso a la habitación de los padres, pero también ocurre con la vegetación, que tanto físicamente como de forma recreada a través de unas alfombras, colonizan el gran salón principal. Una clara obstinación por naturalizar el artificio de la construcción, sincerada en todo caso en el encuentro de lo construido con las dunas mediante unas escaleras, que puede también observarse, persiguiendo sutiles reflejos y coincidencias cromáticas con el entorno. Los vestuarios también dan ejemplo de esto del uso de estos mecanismos. Tras ser expulsados del interior de la casa a petición de los propietarios, prácticamente también lo hicieron de la plataforma. De algún modo, construyendo en un diálogo intencionadamente productivo de la arquitectura con un paisaje artificial que, naturalizándose poco a poco va ampliando sus límites, hasta colonizar sutilmente la esencia del lugar.

\section{Bajo, pero sobre el suelo}

El tercer análisis, observa el vacío de $160 \mathrm{~m}^{2}$, que Aranda, Pigem y Vilalta proponen como umbral entre el interior y exterior del Parc de Pedra Tosca. Se trata de un espacio triangular creado a partir de tres accesos distintos. Para llegar a él, es necesario atravesar un camino dispuesto "sobre el suelo" que va acercándose custodiado por dos muros de contención sin perder cota. Los muros están formados por un gran montículo de piedras volcánicas acumuladas y soportadas sobre sido encastadas en el terreno. La precisión geométrica del corte, la misma artificial presencia del hierro y la a veces puntual disposición discordante con los muros preexistentes del emplazamiento, delatan la voluntad de querer inaugurar una nueva temporalidad en este durante tantos años paisaje olvidado. "Bajo el suelo", pero aún "sobre el suelo", en la misma cota, la perspectiva que se abre es realmente distinta. Además, facilita la observación de varios detalles en verdadera magnitud. En primer lugar, aparece una especie de artiga, atrapada por una secuencia de acero que sustenta un cúmulo de piedras. Las láminas de acero se muestran separadas unos centímetros, con 5-10 grados de ladeo. La configuración del elemento constructivo hace revivir la memoria de las juntas vacías sin argamasas, y la mínima inclinación de soporte por gravedad que exhiben las cercas y caminos del entorno. Desaparece la idea de corte, realmente realizado en el terreno, reconociéndose de forma verosímil el esfuerzo de haber apartado a lado y lado del vacío cada una de las piedras que allí se muestran acumuladas por encima de las láminas de acero. En realidad, lo que hicieron los campesinos en la desamortización de Mendizábal para poder trabajar estas tierras, es lo que ahora provoca reflexionar sobre cómo reactivar este viejo territorio cargado de viejas historias olvidadas.

\section{Sobre, pero bajo el suelo}

El cuarto análisis estudia la fisura de 140m con la que Miralles-Pinós, siguiendo una pendiente pronunciada, construyen el acceso principal a la plaza de sepulturas del Parc Cementiri d'Igualada. La hendidura tiene una sección longitudinal en forma de cuña. El recorrido, parte "sobre el suelo" perdiendo inmediatamente cota en un camino de 6-7m de ancho. Un conjunto de nichos formalizados a través de unas cajas prefabricadas de hormigón, que tienen la función de contención de las tierras existentes en ambos lados del camino, acompañan siempre en paralelo el descenso. La propia presencia del hormigón, la misma intrigante antinatural pendiente de los nichos o la manera de atrapar unos muros de piedra seca, entre mallas electrosoldadas, que ataluzan las pendientes del terreno, delatan también en esta ocasión el origen artificioso de la hendidura. El extremo opuesto de la sección, sin embargo, hace variar la percepción. A pesar de haber descendido varios metros, la sensación es que el cuerpo sigue estando "sobre el suelo". La realidad es que ha quedado sepultado "bajo el suelo". El suceso viene acompañado de la puesta en tiempo y lugar del artificio. El paisaje que rodea, ahora con más perspectiva, empieza realmente a participar del repentino entierro al cual hemos sido invitados. Los taludes empiezan a mostrarse como si hubieran sido esculpidos por las mismas cárcavas de erosión adyacentes; las famosas margas grises de Igualada. Los propios panteones se esfuerzan repetidamente en mostrar un lenguaje primitivo, que resulta próximo a las grutas de las montañas cercanas. Los árboles que fueron encontrados durante el descenso, volviendo al punto de partida sobre los mismos pasos, ahora parecen estar curando, de forma natural, la herida causada en el terreno. Incluso el mismo artificio, en una búsqueda de imperfección consciente de los arquitectos, se desvela participando con naturalidad, de la erosión que provoca el paso del tiempo.

1 BASSEGODA, Juan. Historia de arquitectura. Barcelona: Editores técnicos asociados, SA, 1984, p.19-20.

${ }^{2}$ RICOEUR, Paul. Tiempo y narración I. Configuración del tiempo en el relato histórico. México DF: Siglo XXI Editores, 1995. ${ }^{3}$ LÓPEZ COTELO, Borja. Sverre Fehn: desde el dibujo. Representación e Teoría Arquitectónica, La Coruña, 2012. ${ }^{4}$ HEIDEGGER, Martin. Construir, Habitar, Pensar. Barcelona:
ETSAB-UPC,1995.

5HOLL, Steven. Relaciones complejas, "Edge of a city", Pamphlet Architecture, Nueva York, 1991.

${ }^{6} \mathrm{HOLL}$, Steven. Entrelazamientos. Gustavo Gili, Barcelona,

'MADERUELO, Javier. El paisaje. Génesis de un concepto. Madrid: Abada Editores, 2005.

${ }^{8}$ MERLEAU-PONTY, M. Entre lo visible y lo invisible. Barcelona: Seix Barral, 1970 